\title{
SUPPORTING INFORMATION FOR INTERACTION OF PHOSPHATE WITH LITHIUM COBALT OXIDE NANOPARTICLES: A COMBINED SPECTROSCOPIC AND CALORIMETRIC STUDY
}

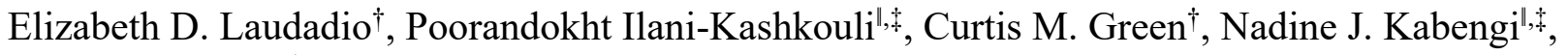 \\ Robert J. Hamers ${ }^{\dagger}$
}

$\dagger$ Department of Chemistry, University of Wisconsin, Madison, WI 53706

"Department of Chemistry, Georgia State University, Atlanta, GA 30303

${ }^{\ddagger}$ Department of Geosciences, Georgia State University, Atlanta, GA 30303

* To whom correspondence should be addressed: rjhamers@wisc.edu

1.0 Extended Methods

1.1 General.

1.2 Unit conversion for amount phosphate adsorbed to $\mathrm{LiCoO}_{2}$ between two methods.....................S2

2.0 Additional Figures

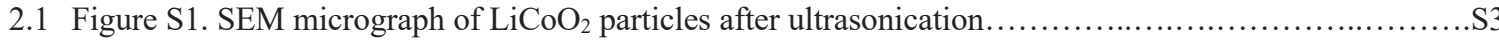

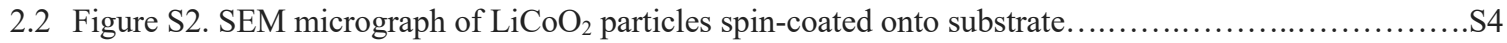

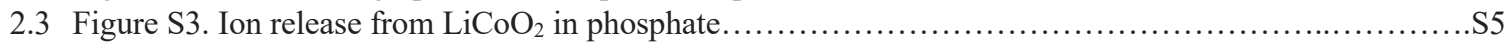

2.4 Figure S4. Representative XPS spectra corresponding to coverage analysis.............................. 6

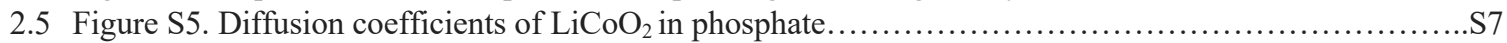

2.6 Figure S6. Time-course ATR-FTIR spectra of phosphate exposure to $\mathrm{LiCoO}_{2} \ldots \ldots \ldots \ldots \ldots \ldots \ldots \ldots \ldots . . . \ldots \ldots \ldots$

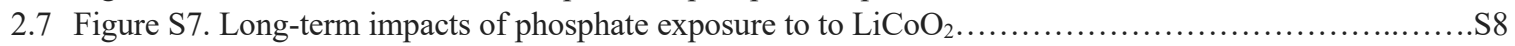




\title{
SUPPORTING INFORMATION FOR INTERACTION OF PHOSPHATE WITH LITHIUM COBALT OXIDE NANOPARTICLES: A COMBINED SPECTROSCOPIC AND CALORIMETRIC STUDY
}

\author{
Elizabeth D. Laudadio $^{\dagger}$, Poorandokht I. Kashkouli ${ }^{\prime \prime}$, Curtis M. Green ${ }^{\dagger}$, Nadine J. Kabengil,\$, \\ Robert J. Hamers ${ }^{\dagger}$
}

${ }^{\dagger}$ Department of Chemistry, University of Wisconsin, Madison, WI 53706

" Department of Chemistry, Georgia State University, Atlanta, GA 30303

${ }^{\ddagger}$ Department of Geosciences, Georgia State University, Atlanta, GA 30303

* To whom correspondence should be addressed: rjhamers@wisc.edu

1.0 Extended Methods.

1.1 General. A Thermo Scientific Sorvall Legend X1R Centrifuge with a Thermo TX-400 rotor at $4696 \mathrm{~g}$ was used for all centrifugation described, unless otherwise noted. All reagents were purchased from Sigma-Aldrich. Ultrapure water (18.2 $\mathrm{M} \Omega / \mathrm{cm}$ resisitivity) was used in all experiments.

1.2 Unit conversion from amount adsorbed via calorimetry to units used for surface coverage in XPS.

$$
\frac{\mu \mathrm{mol}}{\mathrm{mg} \mathrm{LCO}} P(\text { from calorimetry }) \times 10 \mathrm{mg} \mathrm{LCO} \times \frac{1 \mathrm{~mol}}{10^{6} \mu \mathrm{mol}} \times \frac{6.022 \times 10^{23} \text { atoms } P}{1 \mathrm{~mol}}=\text { atoms } P
$$

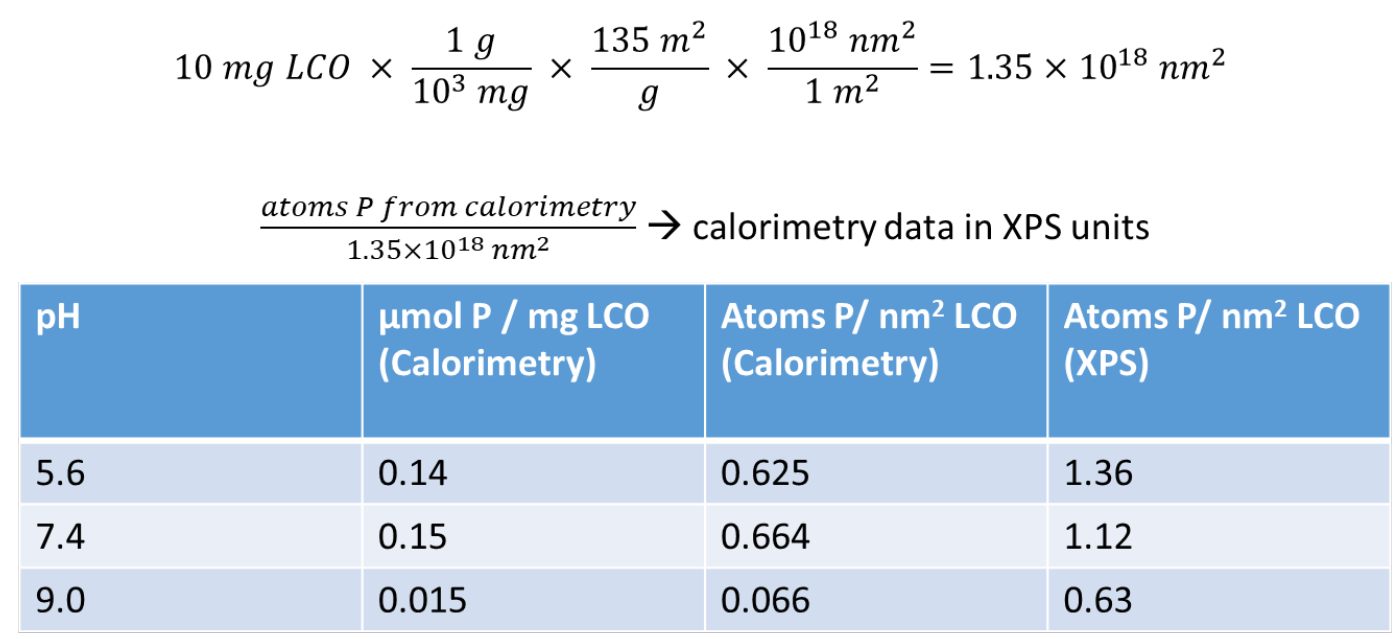


2.0 Additional figures.

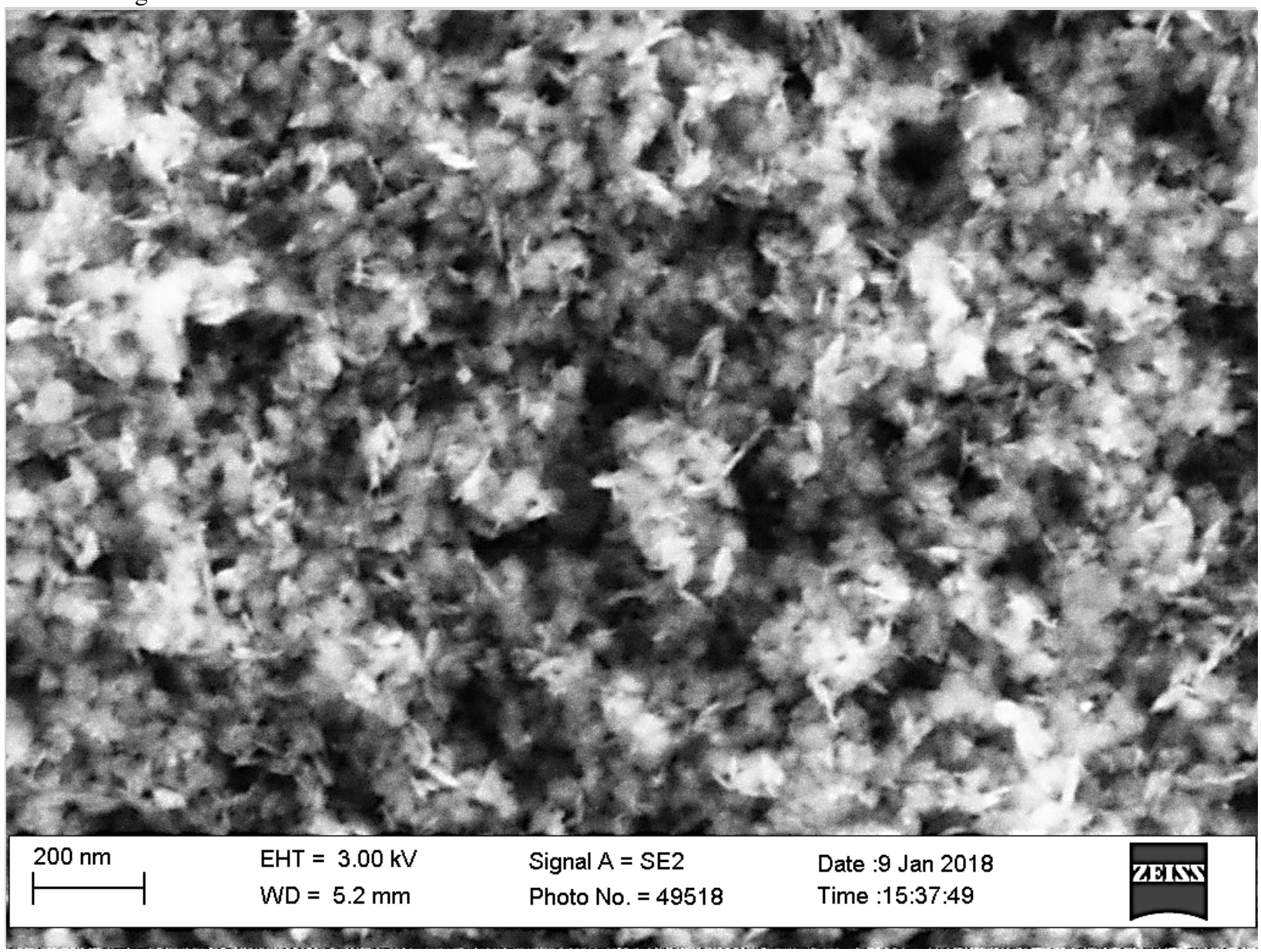

Figure S1. SEM micrograph of $\mathrm{LiCoO}_{2}$ particles following ultrasonication described for DLS measurements and formation of nanoparticle layers for ATR-FTIR. Individual particles are still observed, and particles do not appear to have been fractured in the process. 


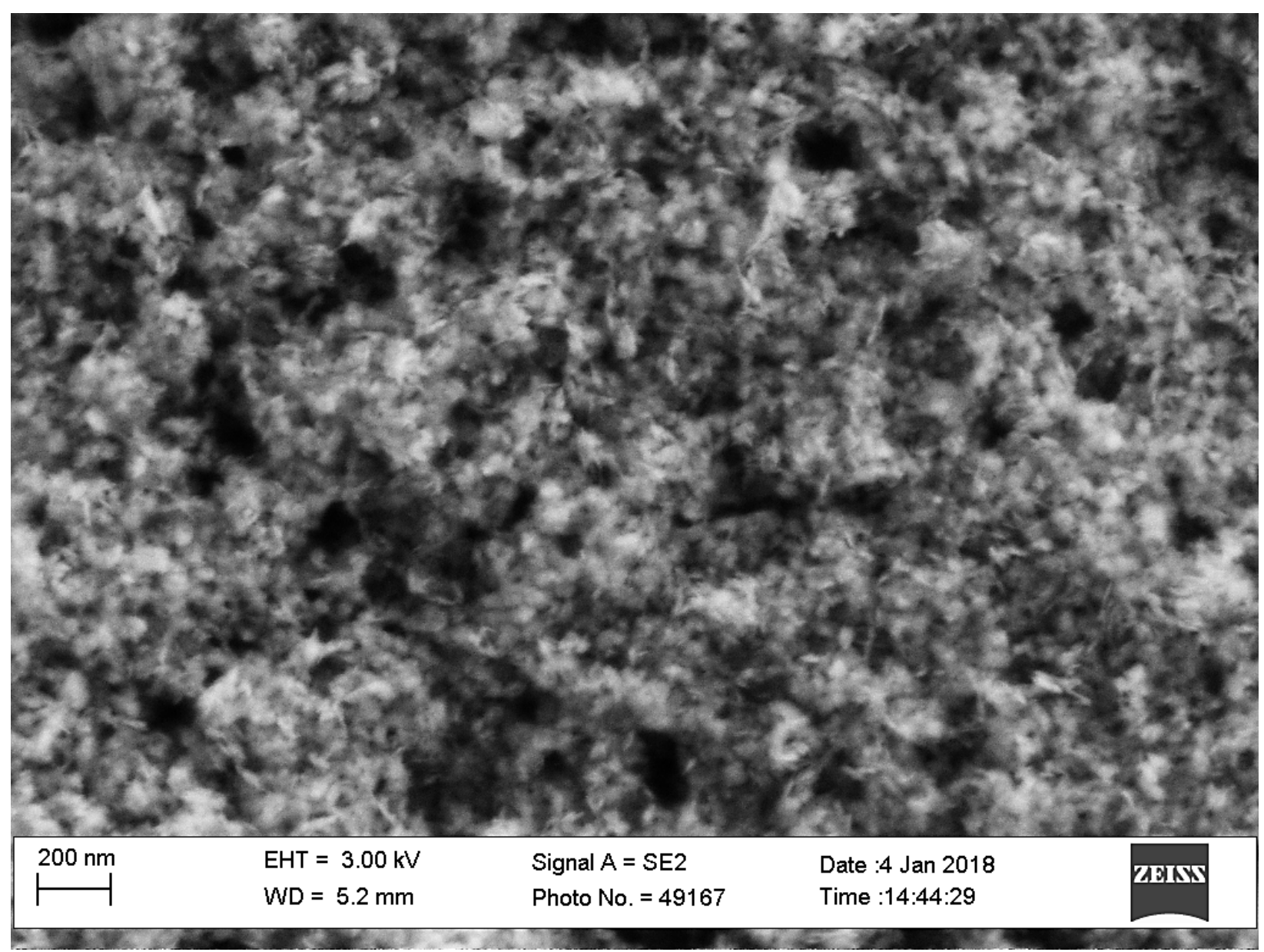

Figure S2. SEM shows $\mathrm{LiCoO}_{2}$ particles randomly orient on surfaces when applied using a spin-coater. 


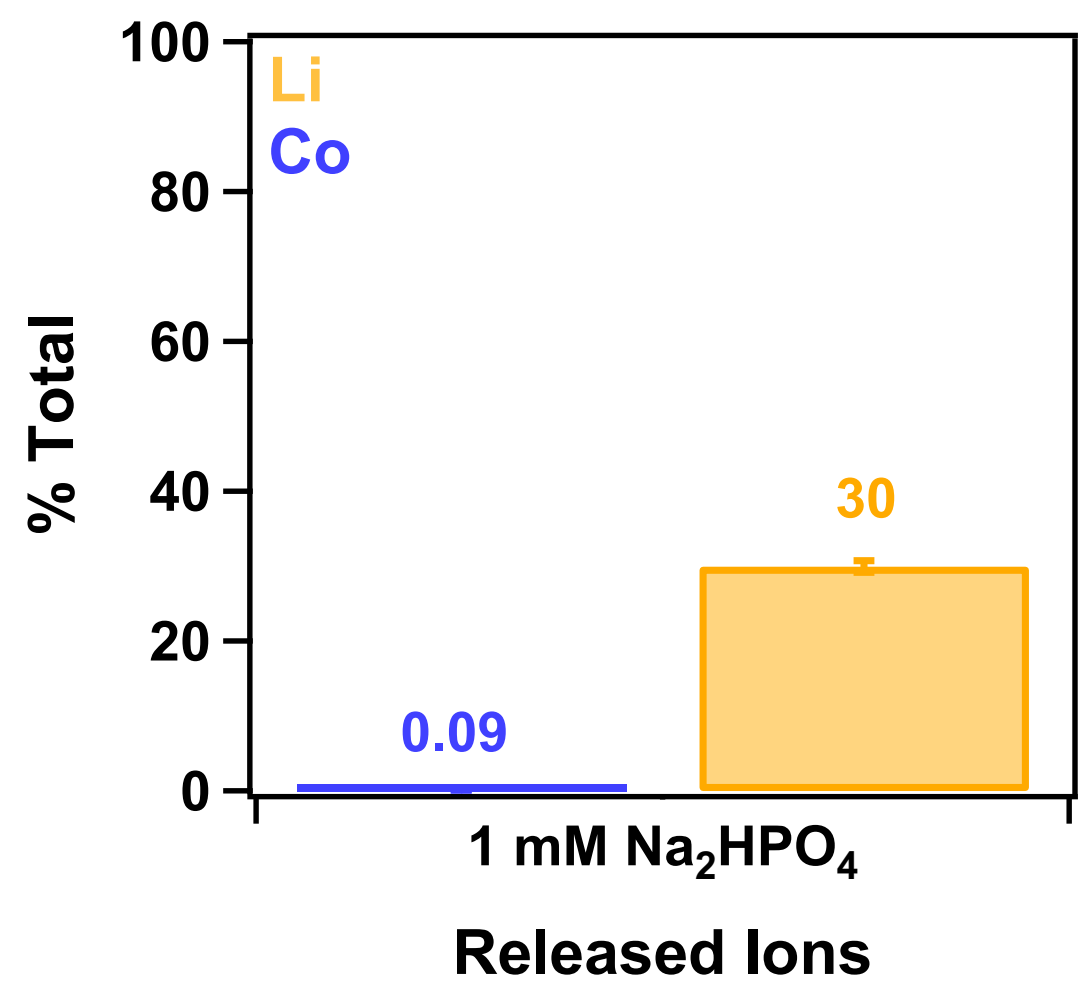

Figure S3. Percentage of total cobalt (blue) and lithium (yellow) ions in the material that are released from a $100 \mathrm{mg} / \mathrm{L}$ suspension of $\mathrm{LiCoO}_{2}$ in $1 \mathrm{mM} \mathrm{Na} \mathrm{HPO}_{4}$ for 1 hour as measured with ICP-MS. 
pH 5.6
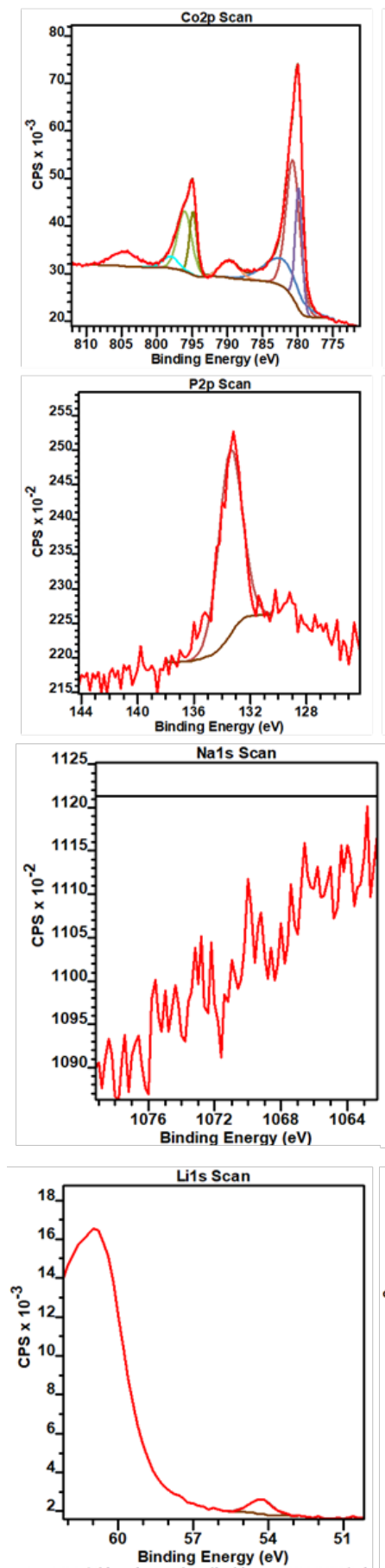

$\mathrm{pH} 7.4$
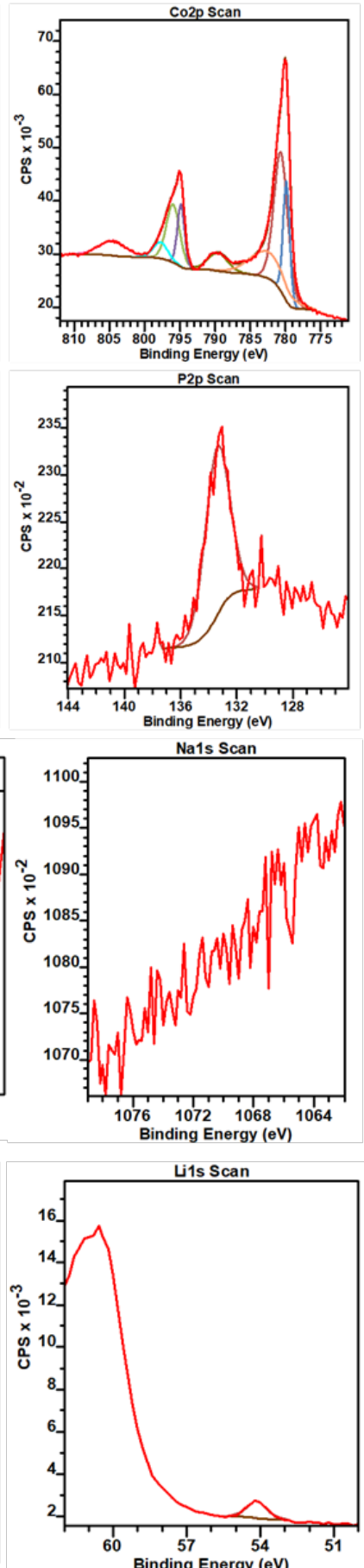

$\mathrm{pH} 9.0$
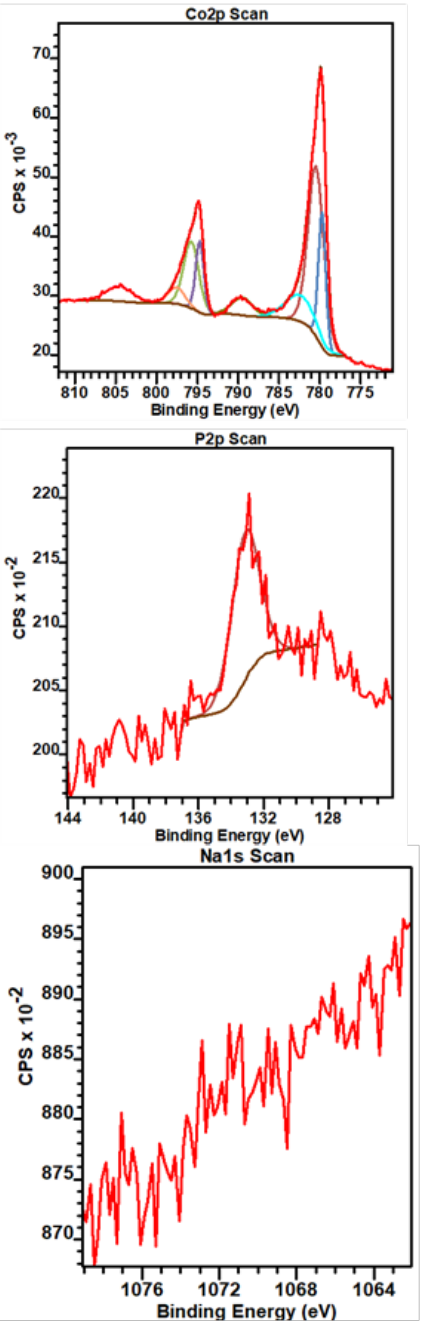

Li1s Scan

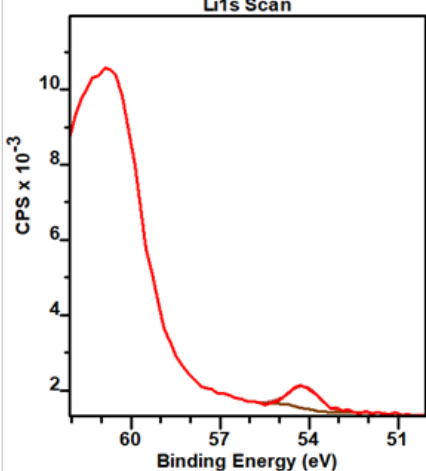

Figure S4. Representative XPS spectra used in the analysis of phosphate coverage on LCO as a function of $\mathrm{pH}$. There is no evidence for sodium binding to the surface. Lithium is observed in each case, but is not used for quantification due to the likelihood of $\mathrm{Li}^{+} / \mathrm{H}^{+}$exchange at the surface in water. 


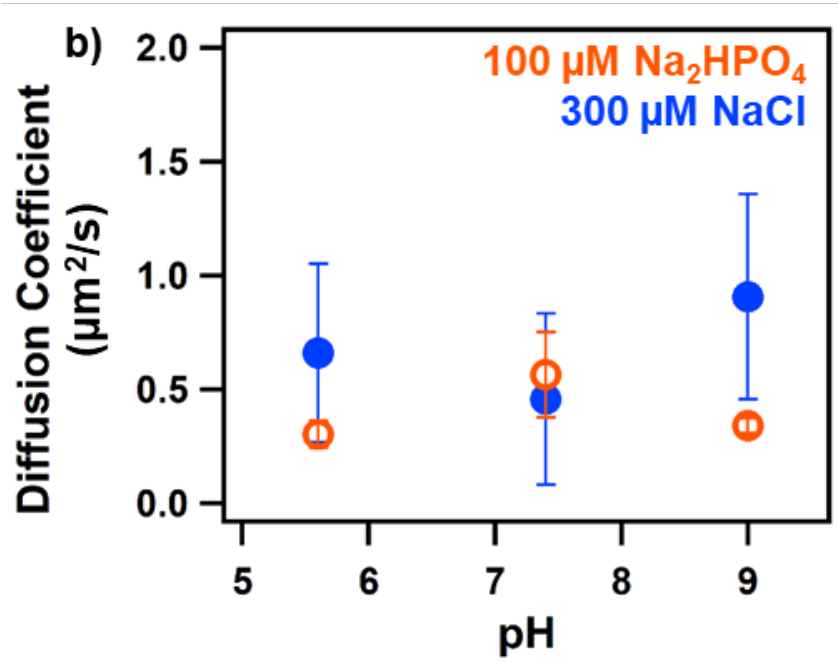

Figure S5. Diffusion coefficient of $\mathrm{LiCoO} 2$ nanoparticles as a function of $\mathrm{pH}$ in the absence and presence of phosphate.

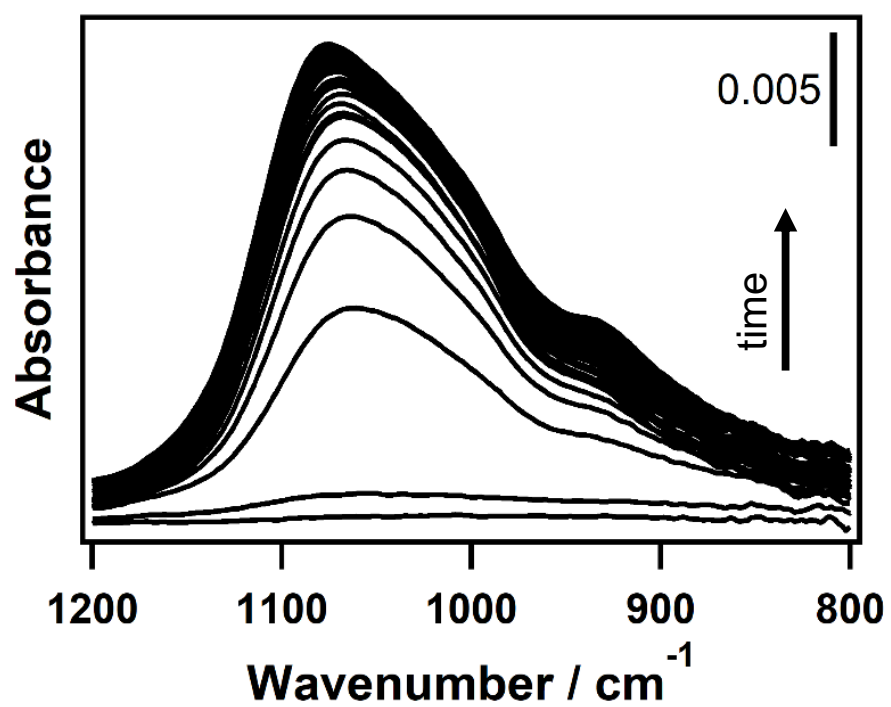

Figure S6. ATR-FTIR spectra collected over the course of one hour of $\mathrm{Na}_{2} \mathrm{HPO}_{4}$ exposure to $\mathrm{LiCoO}_{2}$ at $\mathrm{pH} 7.4$. 

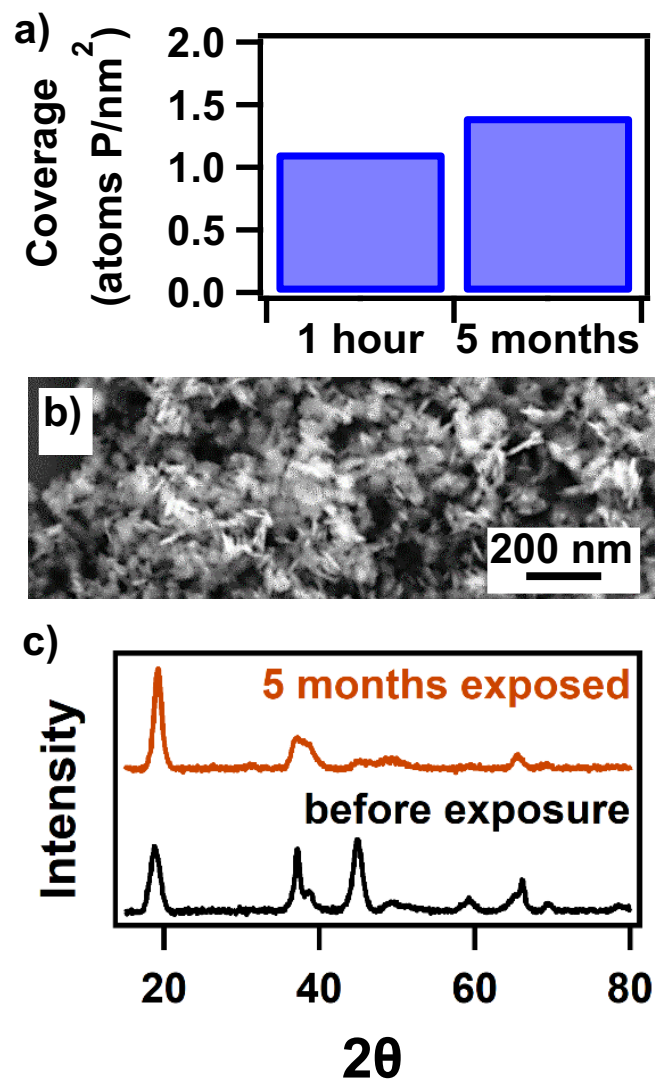

Figure S7. Long term impacts of $\mathrm{Na}_{2} \mathrm{HPO}_{4}$ exposure to $\mathrm{LiCoO}_{2}$ at $\mathrm{pH}$ 7.4. a) coverage values as determined by XPS in atoms $\mathrm{P} / \mathrm{nm}^{2}$ after one hour vs after five months. b) SEM micrograph of $\mathrm{LiCoO}_{2}$ nanosheets after five months in $\mathrm{Na}_{2} \mathrm{HPO}_{4}$ at $\mathrm{pH}$ 7.4. c) XRD patterns of $\mathrm{LiCoO}_{2}$ nanoparticles before exposure (black trace) and after five months exposure to $\mathrm{Na}_{2} \mathrm{HPO}_{4}$ at $\mathrm{pH} 7.4$ (orange trace). 